\title{
Relationship between Metabolic Syndrome and Hearing Loss: Korea National Health and Nutritional Survey
}

\author{
Jiyoung Kim, In Young Cho, Yohwan Yeo, Yun-Mi Song* \\ Department of Family Medicine, Samsung Medical Center, Sungkyunkwan University School of Medicine, Seoul, Korea
}

Background: We aimed to evaluate the association between metabolic syndrome (MetS) and hearing loss (HL) in light of noise exposure in Korean middle-aged adults.

Methods: Subjects were 10,356 adults (4,509 males, 5,847 females) aged 40-80 years, who completed audiometric tests and laboratory examinations as part of the Korea National Health and Nutrition Examination Survey between 2010 and 2012. MetS was defined according to the National Cholesterol Education Program-Third Adult Treatment Panel (NCEP III) and International Diabetes Federation (IDF). Low-frequency HL was defined as pure tone averages $>25$ decibels $(\mathrm{dB})$ at low frequencies $(0.5,1$, and 2 kilohertz $[\mathrm{kHz}])$. High-frequency HL was defined as pure tone averages $>25 \mathrm{~dB}$ at high frequencies $(3,4$, and $6 \mathrm{kHz}$ ). Odd ratios (OR) and $95 \%$ confidence intervals (CI) of MetS associated with each HL type were estimated using multiple logistic regression analysis after adjusting for covariates and taking into consideration sampling weight.

Results: Thirty eight point one percent and $28.6 \%$ met the MetS by NCEP III and IDF criteria, respectively. Prevalence of HL was $29.3 \%$ and $63.9 \%$ for low- and high-frequency HL, respectively. MetS defined by NCEP III was associated with higher risk of high-frequency HL (OR, 1.35; 95\% CI, 1.05-1.73), while MetS by IDF criteria was not. The interaction by the noise exposure on the MetS and high-frequency HL was not significant (P-interaction=0.100). There was no association between MetS and low-frequency HL, regardless of applied diagnostic criteria for MetS.

Conclusion: Our findings suggest MetS is associated with high-frequency HL in people with exposure to noise.

Keywords: Metabolic Syndrome; Hearing Loss; Noise Exposure; Cross-Sectional Studies

Received: October 19, 2019, Revised: March 13, 2020, Accepted: March 30, 2020

*Corresponding Author: Yum-Mi Song https://orcid.org/0000-0001-9232-5563

Tel: +82-2-3410-2442, Fax: +82-2-3410-0338, E-mail: yunmisong@skku.edu 


\section{INTRODUCTION}

Hearing loss (HL) results in reduced quality of life, and leads to limited communication with others, which may result in impaired social connectivity and exert negative effects on the behavioral and social psychological status of persons affected by HL. Consequently, HL may lower self-esteem and lead to decreased social activities, feelings of isolation, depression and anxiety, and poor quality of life. ${ }^{1,2)}$

The prevalence of HL increases with age. Although HL usually begins in the 30s, people usually start to complain of hearing impairment in their 40-60s when reduced hearing ability occurs around the 1,000 $\mathrm{Hz}$ frequency. Additional hearing difficulty may also occur in the $60 \mathrm{~s}$ because of $\mathrm{HL}$ at low range frequencies due to disease, trauma, and degenerative changes. ${ }^{3)}$ A Korean study reported the prevalence of hearing impairment in Korea increased from $4.4 \%$ in $19-39$ years old to $21.1 \%$ in those $40-64$ years old, and $69.7 \%$ in those over 65 years old. ${ }^{4)}$

Given the prevalence of $\mathrm{HL}$ is expected to further increase due to the progressive aging of the worldwide population, it is important to identify modifiable risk factors of HL. Several factors have been suggested to be associated with the risk of HL. In addition to age, cardiovascular disease risk factors, such as hypertension, hyperglycemia, obesity, and smoking, and noise exposure, are known to be associated with hearing impairment. $^{4-7)}$

Metabolic syndrome (MetS), a constellation of several metabolic components, including abdominal obesity, high blood pressure, elevated fasting glucose, and dyslipidemia, has been suggested as an independent risk factor of HL. ${ }^{8,9)}$ In an observational study of Korean women in their 50s or older, high-frequency HL was more prevalent among people with MetS than those without MetS. ${ }^{10)}$ In a study of middle-aged Korean men and women, MetS was associated with both low- and high-frequency hearing impairment in males; although, only hyperglycemia was significantly associated with low- and high-frequency hearing impairment in females. ${ }^{11)}$

On the other hand, studies have reported controversial findings on the association between HL and MetS. Findings of a Korean study revealed the incidence of HL was significantly higher in subjects who had four or more MetS components, but not in subjects who had three or less components, compared with those who had $0-2 .{ }^{12)}$ In addition, a study using the 2010-2012 Korea National Health and Nutrition Examination Survey (KNHANES) reported that MetS was not associated with hearing impairment. ${ }^{13)}$ Differences in demographic characteristics of study subjects and consideration of noise exposure history might have caused the inconsistency of findings between these earlier studies.

Therefore, we conducted this study to investigate the association between MetS and HL in people aged 40 years or older with consideration of noise exposure history.

\section{METHODS}

\section{Study Population}

The study design was cross-sectional using data from the fifth KNHANES conducted between 2010 and 2012. Of the 25,534 subjects who had completed pure tone audiometry, laboratory, and physical examinations during their participation in the fifth KNHANES, we excluded 11,873 who were less than 40 years old. We further excluded participants with any of the following criteria: missing required data for MetS diagnosis (waist circumference, blood pressure, fasting blood sugar, triglyceride, high-density lipoprotein cholesterol [HDL-C]), diagnosed with or receiving treatment for otitis media, using hearing aids or cochlear implants, abnormality found in ear inspection, external ear canal stenosis or eardrum abnormality found during $0^{\circ}$ endoscope examination, or if they reported a restriction in daily life and social activities due to hearing problems. Thus, 10,356 subjects were included in the final analysis (Supplementary Table 1).

\section{Collection of Study Data}

Subject characteristics were collected using a standardized questionnaire, including age, household income, education level, smoking, alcohol consumption, physical activity, history of noise exposure, and use of ear protection. Subjects were categorized into several groups according to the distribution of collected variables. Household income was categorized into five groups: upper, upper-middle, middle, lowermiddle, or lower class. Highest achieved public education level was categorized into four groups: university, high school, middle school, or elementary school. Smoking consumption was categorized into three groups: nonsmokers for subjects who were not smoking at the present and whose lifetime smoking amount was less than 5 packs (100 cigarettes), ex-smokers for subjects who had smoked more than 5 packs during their lifetime but had quit smoking, and current smokers for subjects who smoked currently regardless of the amount of smoking. Alcohol consumption was categorized into four groups depending on frequency of drinking during the recent year: non-drinkers for those who did not drink alcohol during their lifetime or who drank less than once a month for the last 1 year, drinking once a week, drinking 2-3 times a week, or drinking more than 4 times a week. Regular physical activity group was defined as subjects who satisfied any one of the following conditions: (1) vigorous physical activity for more than $20 \mathrm{~min}$ utes, more than 3 days per week, (2) moderate physical activity for more than 30 minutes, more than 5 days per week, or (3) walking for more than 30 minutes, more than 5 days a week.

Study subjects were further categorized into two groups based on noise exposure experience: If a person reported exposure to workplace noise, other place (extra-work) noise, or sudden loud noise, then she/ he was categorized into the noise exposure group. Workplace noise exposure was defined as an affirmative answer to the question regarding whether they had worked for more than 3 months in a place with high noise such as a mechanical or generator sound. Other place noise exposure was defined as having experienced exposure to loud noises for 
more than 5 hours a week. Sudden loud noise exposure was defined as exposure to noise such as a gunshot or explosions.

Height was measured with a SECA 225 (SECA, Hamburg, Germany), and weight was measured with a GL-6000-20 (G-Tech International, Uijungbu, Korea) at intervals of $0.1 \mathrm{~kg}$. Body mass index was calculated by dividing weight $(\mathrm{kg})$ by squared height $\left(\mathrm{m}^{2}\right)$. Waist circumference was measured with a SECA 200 (SECA) at the midpoint between the bottom of the last rib and the top of the iliac crest, after expiratory breathing.

Four nurses from the Specialized Survey Team of the Disease Control Headquarters who oversaw and performed blood pressure measurements. Blood pressure was measured 3 times at an interval of 30 sec-

Table 1. Distribution of metabolic components and selected variables according to frequency-specific hearing loss

\begin{tabular}{|c|c|c|c|c|c|c|c|}
\hline \multirow{2}{*}{ Variable } & \multirow{2}{*}{ Category } & \multicolumn{3}{|c|}{ Low frequency hearing loss } & \multicolumn{3}{|c|}{ High frequency hearing loss } \\
\hline & & No $(\mathrm{N}=7,326)$ & Yes $(N=3,030)$ & P-value & No $(N=3,740)$ & Yes $(N=6,616)$ & P-value* \\
\hline \multicolumn{8}{|l|}{ Metabolic syndrome } \\
\hline \multirow[t]{2}{*}{ By IDF } & No & $5,405(73.1)$ & $1,990(26.9)$ & $<0.001$ & $2,877(38.9)$ & $4,518(61.1)$ & $<0.001$ \\
\hline & Yes & $1,921(64.9)$ & $1,040(35.1)$ & & $863(29.1)$ & 2,098 (70.9) & \\
\hline \multirow{2}{*}{ By NCEP III } & No & $4,773(74.4)$ & $1,639(25.6)$ & $<0.001$ & $2,650(41.3)$ & $3,762(58.7)$ & $<0.001$ \\
\hline & Yes & $2,553(64.7)$ & $1,391(35.3)$ & & $1,090(27.6)$ & $2,854(72.4)$ & \\
\hline \multirow[t]{2}{*}{ Abdominal obesity ${ }^{\dagger}$} & No & $4,412(73.1)$ & $1,624(26.9)$ & $<0.001$ & $2,287(37.9)$ & $3,749(62.1)$ & $<0.001$ \\
\hline & Yes & $2,914(67.5)$ & $1,406(32.5)$ & & $1,453(33.6)$ & $2,867(66.4)$ & \\
\hline \multirow[t]{2}{*}{ Increased blood pressure } & No & $3,850(78.5)$ & $1,054(21.5)$ & $<0.001$ & $2,300(46.9)$ & $2,608(53.1)$ & $<0.001$ \\
\hline & Yes & $3,476(63.8)$ & $1,976(36.2)$ & & $1,444(26.5)$ & $4,008(73.5)$ & \\
\hline \multirow[t]{2}{*}{ Increased fasting glucose } & No & $4,773(73.7)$ & $1,703(26.3)$ & $<0.001$ & $2,661(41.1)$ & $3,815(58.9)$ & $<0.001$ \\
\hline & Yes & $2,553(65.8)$ & $1,327(34.2)$ & & $1,079(27.8)$ & $2,801(72.2)$ & \\
\hline \multirow[t]{2}{*}{ Increased triglyceride } & No & $4,916(70.8)$ & 2,026 (29.2) & 0.814 & $2,678(38.6)$ & $4,264(61.4)$ & $<0.001$ \\
\hline & Yes & $2,410(70.6)$ & $1,004(29.4)$ & & $1,062(45.5)$ & 2,352 (68.9) & \\
\hline \multirow[t]{2}{*}{ Low HDL-C } & No & $4,243(72.6)$ & $1,599(27.4)$ & $<0.001$ & $2,187(37.4)$ & $3,655(62.6)$ & 0.001 \\
\hline & Yes & $3,083(68.3)$ & $1,431(31.7)$ & & $1,553(34.4)$ & $2,961(65.6)$ & \\
\hline Household income & & & & $<0.001$ & & & $<0.001$ \\
\hline Lowest & & $1,365(51.4)$ & $1,289(48.6)$ & & $446(16.8)$ & 2,208 (83.2) & \\
\hline Lower-middle & & $1,370(71.5)$ & $546(28.5)$ & & 657 (34.3) & $1,259(65.7)$ & \\
\hline Middle & & $1,432(76.2)$ & 447 (23.8) & & $764(40.7)$ & $1,115(59.3)$ & \\
\hline High-middle & & $1,344(80.0)$ & $335(20.0)$ & & $787(46.9)$ & $892(53.1)$ & \\
\hline Highest & & $1,721(82.4)$ & $368(17.6)$ & & $1,044(50.0)$ & $1,045(50.0)$ & \\
\hline Education level & & & & $<0.001$ & & & $<0.001$ \\
\hline Elementary school & & $1,777(52.0)$ & $1,643(48.0)$ & & 577 (16.9) & $2,843(83.1)$ & \\
\hline Middle school & & $1,093(70.9)$ & $449(29.1)$ & & $472(30.6)$ & $1,070(69.4)$ & \\
\hline High school & & 2,499 (81.0) & $586(19.0)$ & & $1,457(47.2)$ & $1,628(52.8)$ & \\
\hline University & & $1,869(87.7)$ & $262(12.3)$ & & $1,198(56.2)$ & $933(43.8)$ & \\
\hline Smoking $^{\ddagger}$ & & & & $<0.001$ & & & $<0.001$ \\
\hline Non-smoker & & $4,440(71.9)$ & $1,736(28.1)$ & & $2,707(43.8)$ & $3,469(56.2)$ & \\
\hline Ex-smoker & & $1,445(66.7)$ & $720(33.3)$ & & $479(22.1)$ & $1,686(77.9)$ & \\
\hline Current smoker & & $1,357(73.8)$ & $483(26.3)$ & & $519(28.2)$ & $1,321(71.8)$ & \\
\hline Alcohol consumption ${ }^{\ddagger}$ & & & & $<0.001$ & & & $<0.001$ \\
\hline Non-drinker & & $3,486(66.9)$ & $1,726(33.1)$ & & $1,873(35.9)$ & $3,339(64.1)$ & \\
\hline$\leq 1 / w k$ & & $2,102(77.3)$ & $616(22.7)$ & & $1,165(42.9)$ & $1,553(57.1)$ & \\
\hline $2-3 /$ wk & & $1,038(77.6)$ & $299(22.4)$ & & $454(34.0)$ & $883(66.0)$ & \\
\hline$\geq 4 / w k$ & & $588(67.4)$ & $284(32.6)$ & & $197(22.6)$ & $675(77.4)$ & \\
\hline \multirow[t]{2}{*}{ Regular physical activity ${ }^{\ddagger}$} & No & $3,982(70.1)$ & $1,701(29.9)$ & $<0.001$ & $2,096(36.9)$ & $3,587(63.1)$ & $<0.001$ \\
\hline & Yes & $3,344(71.6)$ & $1,329(28.4)$ & & $1,644(35.2)$ & $3,029(64.8)$ & \\
\hline \multicolumn{8}{|l|}{ Noise exposure } \\
\hline Workplace noise exposure & & $914(71.0)$ & $374(29.0)$ & 0.852 & $373(29.0)$ & $915(71.0)$ & $<0.001$ \\
\hline Protection for workplace noise ${ }^{\S}$ & & $178(74.8)$ & $60(25.2)$ & 0.516 & $73(30.7)$ & $165(69.3)$ & 0.935 \\
\hline Other place noise exposure & & $132(69.8)$ & $57(30.2)$ & 0.784 & $64(33.9)$ & $125(66.1)$ & 0.515 \\
\hline Sudden loud noise exposure & & $1,462(69.5)$ & $643(30.5)$ & 0.146 & $527(25.0)$ & $1,578(75.0)$ & $<0.001$ \\
\hline Protection for sudden loud noisell & & $170(74.6)$ & $58(25.4)$ & 0.290 & $59(25.9)$ & $169(74.1)$ & 0.757 \\
\hline
\end{tabular}

Values are presented as number (\%).

IDF, International Diabetes Federation; NCEP, National Cholesterol Education Program's Adult Treatment Panel III; HDL-C, high-density lipoprotein cholesterol.

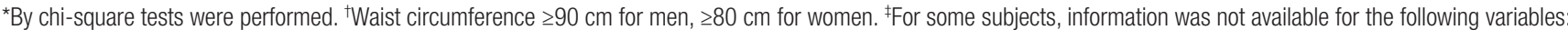
body mass index ( $n=14)$, household income $(n=139)$, education level $(n=178)$, smoking $(n=175$, alcohol consumption $[n=217])$, and regular physical activity $(n=178)$. ${ }^{\S}$ Applicable to the subjects who reported to have experienced workplace noise exposure. "Applicable to the subjects who reported to have experienced sudden loud noise exposure. 
onds, and the mean of the second and third blood pressures was used.

After participants fasted for at least 12 hours, a laboratory test for low-density lipoprotein cholesterol, HDL-C, triglyceride, total cholesterol, and fasting plasma glucose was performed.

\section{Audiometric Measure and Definition of Hearing Loss}

Skilled otorhinolaryngology residents conducted participant ear examinations. Pure-tone audiometry was performed using an automated diagnostic audiometer (SA 203; Entomed, Malmo, Sweden) at 6 frequencies $(0.5,1,2,3,4$, and 6 kilohertz $[\mathrm{kHz}])$ in a double-walled audiovisual booth where noises were interrupted.

In this study, we defined low-frequency $\mathrm{HL}$ as pure tone averages at three low frequencies $(0.5,1$, and $2 \mathrm{kHz})$ exceeding 25 decibels (dB) unilaterally and or bilaterally. High-frequency HL were defined as pure tone averages at three high frequencies $(3,4$, and $6 \mathrm{kHz})$ exceeding $25 \mathrm{~dB}$ unilaterally and/or bilaterally., ${ }^{4,13)}$

\section{Definition of Metabolic Syndrome}

We applied two MetS criteria recommended by the 2005 revised National Cholesterol Education Program's III (NCEP III) and by the International Diabetes Federation (IDF). NCEP III criteria for Asia was published by the National Heart, Lung and Blood Institute of the National Institute of Health and it requires the presence of at least three of the following components: (1) abdominal obesity (waist circumference $\geq 90 \mathrm{~cm}$ for male, $\geq 80 \mathrm{~cm}$ for female), (2) taking antihypertensive medication or systolic blood pressure (SBP) $\geq 130 \mathrm{~mm} \mathrm{Hg}$ or diastolic blood pressure (DBP) $\geq 85 \mathrm{~mm} \mathrm{Hg}$, (3) fasting plasma glucose (FPG) $\geq 100$ $\mathrm{mg} / \mathrm{dL}$ or hemoglobin $\mathrm{Alc}(\mathrm{HbAlc}) \geq 6.5 \%$ or receiving drug treatment for elevated FPG, (4) triglyceride $\geq 150 \mathrm{mg} / \mathrm{dL}$, or (5) HDL cholesterol $\leq 40 \mathrm{mg} / \mathrm{dL}$ for male, $\leq 50 \mathrm{mg} / \mathrm{dL}$ for female.

The IDF criteria includes abdominal obesity (waist circumference $\geq 90 \mathrm{~cm}$ for male, $\geq 80 \mathrm{~cm}$ for female) as a prerequisite for the identification of MetS. In addition to abdominal obesity, two or more of the following four factors should exist: (1) SBP $\geq 130 \mathrm{~mm} \mathrm{Hg}$ or DBP $\geq 85 \mathrm{~mm}$ Hg or taking antihypertensive medication; (2) FPG $\geq 100 \mathrm{mg} / \mathrm{dL}$ or HbAlc $\geq 6.5 \%$ or drug treatment for elevated FPG; (3) triglyceride $\geq 150$ $\mathrm{mg} / \mathrm{dL}$; or (4) HDL cholesterol $\leq 40 \mathrm{mg} / \mathrm{dL}$ for male, $\leq 50 \mathrm{mg} / \mathrm{dL}$ for female.

\section{Statistical Analyses}

Descriptive statistics were used to present the general characteristics of the study subjects. Chi-square tests were performed to evaluate univariate associations of risk factors and MetS with type of HL. Multivariable-adjusted association of MetS and metabolic components with each type (low-frequency, high-frequency) of hearing impairment were evaluated using multiple logistic regression analysis taking into consideration the sampling weights for the Korean standard population. We repeated the multiple logistic regression analyses according to the presence of noise exposure history. Statistical analyses were performed using SAS software ver. 9.4 (SAS Institute Inc., Cary, NC, USA). Significance was set a prior at $\mathrm{P}<0.05$, two-tailed.

\section{Ethics Statement}

The study was approved by the institutional review board of Samsung Medical Center (IRB approval no., SMC 2019-06-102). The informed consent requirement was waived because subjects' consent had been given for the KNHANES. The dataset is in the public domain and does not include individually identifiable information.

\section{RESULTS}

Mean age of study participants was 58.0 (standard deviation=11.1) years, and $49.0 \%$ were male. The prevalence of MetS was $28.6 \%$ by IDF criteria and $38.1 \%$ by NCEP III criteria. Overall, the prevalence of both low-frequency HL $(\mathrm{P}<0.001)$ and high-frequency HL $(\mathrm{P}<0.001)$ tended to increase with age increase (Supplementary Figure 1) in both sexes.

Table 1 shows the distribution of metabolic components and selected variables according to the presence of each HL type. The prevalence of MetS defined by IDF as well as by the NCEP III criteria were significantly $(\mathrm{P}<0.001)$ higher among subjects with $\mathrm{HL}$, regardless of HL type.

Among the MetS components, four factors, except increased triglyc-

Table 2. Association between hearing loss and metabolic syndrome

\begin{tabular}{|c|c|c|c|c|c|c|c|}
\hline \multirow{2}{*}{ Variable } & \multicolumn{2}{|c|}{ Overall subjects $(n=10,356)$} & \multicolumn{2}{|c|}{ Noise exposure group ${ }^{\star}(n=3,217)$} & \multicolumn{2}{|c|}{ No noise exposure group $(n=7,139)$} & \multirow{2}{*}{ P-interaction } \\
\hline & Adjusted OR $(95 \% \mathrm{Cl})^{\dagger}$ & P-value & Adjusted OR $(95 \% \mathrm{Cl})^{\dagger}$ & P-value & Adjusted OR $(95 \% \mathrm{Cl})^{\dagger}$ & P-value & \\
\hline \multicolumn{8}{|c|}{$\begin{array}{l}\text { Association with low-frequency } \\
\text { hearing loss }\end{array}$} \\
\hline MetS by IDF & $0.95(0.83-1.09)$ & 0.472 & $0.90(0.70-1.15)$ & 0.389 & $0.97(0.83-1.13)$ & 0.656 & 0.663 \\
\hline MetS by NCEP III & $1.00(0.88-1.15)$ & 0.952 & $1.00(0.81-1.25)$ & 0.968 & $1.00(0.85-1.16)$ & 0.953 & 0.998 \\
\hline \multicolumn{8}{|c|}{$\begin{array}{l}\text { Association with high-frequency } \\
\text { hearing loss }\end{array}$} \\
\hline MetS by IDF & $1.09(0.94-1.27)$ & 0.256 & $1.30(0.96-1.75)$ & 0.089 & $0.98(0.82-1.17)$ & 0.787 & 0.251 \\
\hline MetS by NCEP III & $1.13(0.99-1.29)$ & 0.073 & $1.35(1.05-1.73)$ & 0.018 & $1.00(0.85-1.18)$ & 0.991 & 0.100 \\
\hline
\end{tabular}

OR, odds ratio; Cl, confidence interval; MetS, metabolic syndrome; IDF, International Diabetes Federation; NCEP III, National Cholesterol Education Program's Adult Treatment Panel III.

* If a person reported to have been exposed to workplace noise, other place (extra-work) noise, or sudden loud noise, he or she was categorized into the noise exposure group. tEstimated by multiple logistic regression analysis after adjusting for sex, age, household income, smoking, alcohol consumption, and physical activity, with consideration of the sampling weight for a multi-stage probability sample design. 
eride level, were associated with both types of HL. Increased triglyceride level was associated with high-frequency HL but not with low-frequency HL.

The higher the household income and education level, the lower the prevalence of both types of HL. Subjects who were involved in regular physical activity had lower rates of both types of HL. Among the items related to noise exposure, workplace noise exposure and sudden loud noise exposure were significantly $(\mathrm{P}<0.001)$ associated with highfrequency HL.

Table 2 shows the multivariable-adjusted associations between MetS and each type of HL. In all subjects, both types of HL were not associated with MetS, regardless of the diagnostic criteria. When we repeated the analysis in two groups stratified by history of noise exposure, any association between low frequency HL and MetS (by NCEP and IDF criteria) was not found regardless of previous noise exposure. However, high-frequency HL (odds ratio, 1.35; 95\% confidence interval, 1.05-1.73) was associated with MetS by NCEP III in the group with noise exposure, although the interaction by the noise exposure on the MetS and high-frequency HL was not significant (P-interaction= $0.100)$.

\section{DISCUSSION}

In this study of Korean males and females over 40 years old, we did not find a significant association between MetS and both types of HL. However, when previous noise exposure was taken into consideration, we found MetS, defined by the NCEP III criteria, was significantly associated with high-frequency HL in the noise exposure group.

Major causes of sensorineural HL are senile HL and noise-induced HL. However, considerable variations in the degree of HL among people with similar age and noise exposure suggest there may be individual specific risk factors that can affect HL. ${ }^{7)}$ Many studies have been conducted to identify the risk of HL associated with cardiovascular factors. However, findings have been inconsistent, probably because HL and cardiovascular diseases could be caused through the same pathologic mechanisms of peripheral vascular injury. Findings of a Korean study suggest several factors, including increased age, tobacco use, education level, hypertension, and low estimated glomerular filtration rate $\left(<60 \mathrm{~mL} / \mathrm{min} / 1.73 \mathrm{~m}^{2}\right)$, are independent risk factors for HL. ${ }^{4)}$ Our study revealed similar findings such that older age, low household income, and smoking were independent risk factors for low- and high-frequency HL.

Considering our study finding that high-frequency HL was associated with MetS only in the group with noise exposure, noise exposure may provoke HL in patients with MetS. Mechanical and metabolic damage caused by noise exposure may further trigger HL in the presence of impaired microcirculation of the cochlear caused by increased viscosity and an increased inflammatory response in persons with MetS.

Advanced age is a well-known risk factor of HL. The reason for increased incidence of HL with increasing age is older people are more likely to have decreased cochlear blood supply and be exposed to risk factors of HL and HL-related illness. ${ }^{14)}$

Several studies have reported that men are more likely compared to women to have high-frequency HL. ${ }^{4,11,15)}$ This may be due to men's greater exposure to occupational noise than women. Estrogen may also play a positive role in protecting hearing function. Loss of the estrogen receptors in the inner ear of men can increase the risk of damage by noise exposure and, thus, HL. ${ }^{16)}$

An inverse correlation has been consistently observed between household income and low- and high-frequency HL, including the current study findings. ${ }^{13,17}$ ) There could be several reasons for this finding. A person with low income might be more likely to be exposed to noisy environments, such as labor or manufacturing. In addition, the low-income group is presumably more affected by risk factors such as smoking. Smoking causes direct vasoconstriction by nicotine and hypoxia through chronic inhalation of carbon monoxide. ${ }^{18-20)}$ In addition, the progression of atherosclerosis by smoking may also exert a negative effect on the inner microcirculation, which may cause hearing impairment. $^{21)}$

This study has several limitations. First, this study is a cross-sectional study, and, therefore, it is difficult to identify causality for the observed association. Second, information on some variables, such as smoking, exercise, and noise exposure were acquired on the basis of self-administered questionnaires, so reporting or recall bias might have influenced the validity of study data, and, hence, study findings. Third, effects using ototoxic drugs that affect HL could not be considered due to the lack of available information.

In conclusion, the findings of the present study suggest MetS is associated with high-frequency HL in people with exposure to noise. However, given the limitation of a cross-sectional study design, prospective studies are needed to clarify the causal relationship between MetS and HL.

\section{CONFLICT OF INTEREST}

No potential conflict of interest relevant to this article was reported.

\section{SUPPLEMENTARY MATERIALS}

Supplementary materials can be found via https://doi.org/10.4082/ kjfm.19.0131. Supplementary Table 1. Baseline characteristics of study population by noise exposure. Supplementary Figure 1. Prevalence of both low-frequency HL (A) and high-frequency HL (B) according to age and sex.

\section{ORCID}

Jiyoung Kim: https://orcid.org/0000-0002-6739-3545

In Young Cho: https://orcid.org/0000-0002-6240-9026

Yohwan Yeo: https://orcid.org/0000-0002-1379-0510

Yun-Mi Song: https://orcid.org/0000-0001-9232-5563 


\section{REFERENCES}

1. Choi J, Chung WH. Age-related hearing loss and the effects of hearing aids. J Korean Med Assoc 2011;54:918-24.

2. Ciorba A, Bianchini C, Pelucchi S, Pastore A. The impact of hearing loss on the quality of life of elderly adults. Clin Interv Aging 2012; 7:15963.

3. Kim SH, Yeo SG. Presbycusis. Hanyang Med Rev 2015;35:78-83.

4. Hong JW, Jeon JH, Ku CR, Noh JH, Yoo HJ, Kim DJ. The prevalence and factors associated with hearing impairment in the Korean adults: the 2010-2012 Korea National Health and Nutrition Examination Survey (observational study). Medicine (Baltimore) 2015;94:e611.

5. Lee JS, Kim DH, Lee HJ, Kim HJ, Koo JW, Choi HG, et al. Lipid profiles and obesity as potential risk factors of sudden sensorineural hearing loss. PLoS One 2015;10:e122496.

6. Yikawe SS, Iseh KR, Sabir AA, Inoh MI, Solomon JH, Aliyu N. Cardiovascular risk factors and hearing loss among adults in a tertiary center of Northwestern Nigeria. World J Otorhinolaryngol Head Neck Surg 2017;4:253-7.

7. Kim JS, Yeh MH, Chun BY, Woo KH, Kang YS, Kim KY, et al. Effect of cigarette smoking on air-conduction hearing threshold level in adult men. Korean J Prev Med 1998;31:285-92.

8. Aghazadeh-Attari J, Mansorian B, Mirza-Aghazadeh-Attari M, Ahmadzadeh J, Mohebbi I. Association between metabolic syndrome and sensorineural hearing loss: a cross-sectional study of 11,114 participants. Diabetes Metab Syndr Obes 2017;10:459-65.

9. Chien CY, Tai SY, Wang LF, Hsi E, Chang NC, Wu MT, et al. Metabolic syndrome increases the risk of sudden sensorineural hearing loss in Taiwan: a case-control study. Otolaryngol Head Neck Surg 2015;153: 105-11.

10. Kim TS, Kim EH, Chung JW. The association between age-related hearing impairment and metabolic syndrome in Korean women: 5-year follow-up observational study. Metab Syndr Relat Disord 2017; 15:240-5.

11. Nam JS, Kim KT, Choi HJ, Yun KE. The relationship between metabolic syndrome and hearing impairment in middle aged men and women. Korean J Fam Pract 2011;1:48-54.

12. Shim HS, Shin HJ, Kim MG, Kim JS, Jung SY, Kim SH, et al. Metabolic syndrome is associated with hearing disturbance. Acta Otolaryngol 2019;139:42-7.

13. Lee HY, Choi YJ, Choi HJ, Choi MS, Chang DS, Kim AY, et al. Metabolic syndrome is not an independent risk factor for hearing impairment. J Nutr Health Aging 2016;20:816-24.

14. Hong SC, Bae Sw, Lee JY. Evaluation of factors affecting sensory neural hearing loss. Korean J Prev Med 1998;31:249-64.

15. Kim S, Lim EJ, Kim HS, Park JH, Jarng SS, Lee SH. Sex differences in a cross sectional study of age-related hearing loss in Korean. Clin Exp Otorhinolaryngol 2010;3:27-31.

16. Hultcrantz M, Simonoska R, Stenberg AE. Estrogen and hearing: a summary of recent investigations. Acta Otolaryngol 2006;126:10-4.

17. Emmett SD, Francis HW. The socioeconomic impact of hearing loss in U.S. adults. Otol Neurotol 2015;36:545-50.

18. Zelman S. Correlation of smoking history with hearing loss. JAMA 1973;223:920.

19. Maffei G, Miani P. Experimental tobacco poisoning: resultant structural modifications of the cochlea and tuba acustica. Arch Otolaryngol 1962;75:386-96.

20. Lacerda A, Leroux T, Morata T. Ototoxic effects of carbon monoxide exposure: a review. Pro Fono 2005;17:403-12.

21. Matschke RG. Smoking habits in patients with sudden hearing loss: preliminary results. Acta Otolaryngol Suppl 1990;476:69-73. 